\title{
Output Factor Measurement of a Dedicated Intra-Operative Radiotherapy (IORT) Accelerator Using Ethanol Chlorobenzene (ECB) Dosimeter and Comparing the Results with Ionometric Dosimetry and Monte Carlo Simulation
}

\author{
Mojtaba Barzegar, ${ }^{1,}$ Ali Reza Shirazi, ${ }^{1}$ Seyed Rabi Mahdavi, ${ }^{2}$ and Hamid Reza Baghani ${ }^{3,4}$ \\ ${ }^{1}$ Medical Physics Department, Tehran University of Medical Sciences, Tehran, Iran \\ ${ }^{2}$ Medical Physics Department, Iran University of Medical Sciences, Tehran, Iran \\ ${ }^{3}$ Radiation Medicine Department, Shahid Beheshti University of Medical Sciences, Tehran, Iran \\ ${ }^{4}$ Physics Department, Hakim Sabzevari University, Sabzevar, Iran \\ "Corresponding author: Mojtaba Barzegar, Medical Physics Department, Tehran University of Medical Sciences, Tehran, Iran. Tel: +98-2122453970, E-mail: \\ tums.radiology@gmail.com
}

Received 2015 September 29; Revised 2016 January 19; Accepted 2016 March 26.

\begin{abstract}
Background: The accurate dose delivery in intraoperative radiotherapy (IORT) tightly depends on the precision of measured dose by the ion chamber. Output factor (OF) measurement of dedicated intraoperative electron radiotherapy (IOERT) accelerators using ion chamber faces some technical challenges including determination of Ksat.

objectives: The goal was to evaluate the performance of ethanol chlorobenzene (ECB) dosimeter in measuring the OF of intraoperative electron beam and to compare the results to those of ionometric dosimetry and Monte Carlo simulation. Consequemtly we determined the Ksat of employed ion chamber through comparison of the ECB response to ion chamber.

Materials and Methods: LIAC dedicated accelerator (LIAC Sordina SpA, Italy) was used for irradiation. To calculate the Ksat, ECB and Advanced Markus chamber were placed at the depth of maximum dose for different energies of LIAC accelerator. Then, Ksat was calculated through comparison of the obtained results. To measure the OF of electron beam, ECB was placed at the depth of maximum dose for each combination of energy/applicator size, and the absorbed dose was determined. Obtained results were compared to those of Advanced Markus chamber and Monte Carlo simulation.

Results: The results of Ksat measurement showed that there is a very good agreement between the practically obtained Ksat and theoretical values determined by Laitano formalism at different energies (the maximum difference between the results was lower than $1 \%$ ). The results of ECB OF measurement were in accordance to the results of ionometric dosimetry and Monte Carlo simulation (the maximum difference between the results was about $1.5 \%$ and $1.7 \%$, respectively).

Conclusion: Based on the results, it may be concluded that the ECB dosimeter could be considered as an accurate tool for both OF measurement of intraoperative electron beam and cross calibration of employed ion chambers for absolute dosimetry (determination of the Ksat).
\end{abstract}

Keywords: Radiation Dosimetry, Ionizing Radiation, Monte Carlo Method

\section{Background}

Intraoperative radiation therapy (IORT) is an innovative treatment technique that delivers a large single fraction of radiation dose to the tumor bed during surgery $(1,2)$. This kind of radiotherapy could be implemented by three different modalities including high-dose rate brachytherapy (HDR-IORT), low kV-IORT and intraoperative electron radiation therapy (IOERT) (3). Due to the limited range of electron beam and short treatment time, IOERT is the main of interest (1).

IOERT is usually performed using some mobile dedicated accelerators that are specifically conceived and designed to work directly in the operating room (OR)(4). Irra- diation is administered on the same operating table where the surgery is carried out (5). One of the most important features of these mobile dedicated radiotherapy units is production of a very high dose per pulse electron beam. The advantages of this radiotherapy modality are sharp dose falloff, ideal dose distribution, short treatment time, high radiobiological effectiveness and normal tissue protection (6).

Today, some types of dedicated accelerators including Mobetron, Novac and LIAC are introduced for IOERT (1). These accelerators are able to produce a high dose per pulse electron beam ranging from 30 to 60 mGy pulse-1 compared to 1-6 mGy pulse-1 for a conventional linear ac- 
celerator (LINAC) (7).

Using the ionization chambers for dose measurements and calibration of high dose per pulse electron beam needs an appropriate method to precisely determine the ion recombination correction factor $\left(\mathrm{K}_{\text {sat }}\right)$ of employed ion chamber (8). Using two voltage analysis (TVA) method, which is recommended by IAEA (Technical report series), TRS-398, and American Association of Physicists in Medicine (AAPM) (Task group) TG-51 protocols causes some degree of uncertainty in $\mathrm{K}_{\mathrm{sat}}$ determination (1, 9-11). This uncertainty in the determination of recombination correction factor of ions formed in the chamber sensitive volume is due to the fact that the free electron fraction created in the chamber sensitive volume is not taken into account during determination of recombination correction factor using the TVA method $(12,13)$. Therefore, the extended Boag theory and Laitano or Di Martino formalism must be applied to determine the recombination correction factor of ion chambers used for calibration of intraoperative radiotherapy electron beams (1,14-16). As an alternative, one can choose a dosimeter that has an independent dose rate response $(17,18)$. In this situation, dosimeter could be employed for absolute and relative dosimetry in high dose per pulse intraoperative electron beam without any further complication. Chemical dosimeters are one of such dosimeters that are recently recommended for output measurements of intraoperative electron beam $(12,19)$.

\section{Objectives}

The aim of this study was to introduce the ethanol chlorobenzene (ECB) chemical dosimeter for output measurements of intraoperative electron beam and comparing the obtained results to those of ionometric dosimetry and Monte Carlo simulation $(20,21)$. Furthermore, the values of Ksat for employed ion chamber were practically determined through cross calibration of ion chamber response relative to the ECB dosimeter response. The determined $\mathrm{K}_{\text {sats }}$ were compared with theoretical values which were calculated by Laitano formalism.

\section{Materials and Methods}

\subsection{LIAC IORT Accelerator}

Irradiations were performed using the mobile dedicated electron accelerator of LIAC (12 MeV model, Sordina, SpA, Italy). It is an intraoperative radiotherapy system, which produces electron with energies of $6,8,10$, and 12 $\mathrm{MeV}$. This accelerator is a newly designed LINAC operating in the $\pi / 2$ mode at $2998 \mathrm{MHz}$, and electron energy is set by varying the radiofrequency power from 1.2 up to $3 \mathrm{MW}$.
Dose rate of this machine could be adjusted from 3 to 40 Gy/min (22).

The accelerating structure, specifically designed for LIAC (12 MeV model) is $92.5 \mathrm{~cm}$ long and consists of 19 accelerating cavities. Radiofrequency power is supplied by an E2V magnetron MG6090 (21). Pulse repetition frequency (PRF) could be varied from 1 to $60 \mathrm{~Hz}$ (1).

Electron beam collimation system consists of two parts: a holder that is attached to the head of the accelerator and an applicator section that is in contact with the patient and holder. The applicators are made of sterillizable PMMA cylindrical tubes with $0.5 \mathrm{~cm}$ thickness and 60 $\mathrm{cm}$ length. The diameter of these applicators changes between 3 and $10(3,4,5,6,7,8$, and 10) $\mathrm{cm}$ and the base angle varies from $0^{\circ}$ to $45^{\circ}\left(0^{\circ}, 15^{\circ}, 30^{\circ}\right.$, and $\left.45^{\circ}\right)$. Dose delivery to the bed tumors is performed through employing the hard docking mechanism. The distance from the scattering foil to the end of the applicator, source to surface distance (SSD) is $713 \mathrm{~mm}$. The passive beam shaping technique allows good uniformity and flatness of the radiation field and a very low $x$-ray contamination $(1,22)$.

\subsection{ECB Dosimetry System}

The ethanol chlorobenzene (ECB) is a type of chemical dosimeter that provides a reliable means for measuring the absorbed dose in different materials (20). It is based on a process of radiolytic formation of hydrochloric acid $(\mathrm{HCl})$ in aqueous ethanolic solutions of chlorobenzene produced by ionizing radiation. This chemical dosimeter has some features such as stability and accuracy of response, ease of production, low price, availability, and independent response to energy, dose, and dose rate. Therefore, this dosimetry system could be a rational choice because of its desirable features (6).

The ECB solution was flame sealed in $2 \mathrm{ml}$ borosilicate glass vials (inner, 9.7; outer, $10.7 \mathrm{~mm}$ in diameter and 37 $\mathrm{mm}$ in length; thickness of glass, $0.5 \mathrm{~mm}$ ) and filled with 24 vol.\% monochlorobenzene, 4 vol.\% distilled water, 0.04 vol.\% acetone, 0.04 vol. \% benzene and 71.92 vol.\% absolute ethanol (20).

To calibrate the ECB dosimeters, a conventional radiotherapy LINAC (PRIMUS, Siemens, Germany) was employed. Irradiations were performed at room temperature (20 to $25^{\circ} \mathrm{C}$ ) in the water phantom and at the reference depth of $8 \mathrm{MeV}$ electron beam in the open field (without any applicator attached to the accelerator head). To reduce the statistical uncertainties associated with the ECB based measurements, four vials were irradiated in phantom for each dose level $(4,8,12,16,20$ and 24 Gy). Finally, the calibration curve was obtained through fitting a straight line to the delivered dose versus ECB response (adjusted $\mathrm{R}^{2}=$ 0.768). 
Then, the calibrated ECB dosimetry system was used in the operating room of Khatam hospital (Tehran, Iran) for dose to water measurements in intraoperative electron beam collimated by specific IORT applicators.

The exposed ECB dosimeters were read at dosimetry laboratory of atomic energy agency of Tehran through optical absorption measurement with a DU 8800 spectrophotometer at the wavelength of $485 \mathrm{~nm}$. The associated uncertainty with the dose measurement was about $1.5 \%(1 \sigma)$.

\subsubsection{Ionometric Dosimetry}

Advanced Markus ion chamber (PTW, Germany) was employed for ionometric dosimetry. Advanced Markus is a vented parallel plate ion chamber with sensitive volume of $0.02 \mathrm{~cm}^{3}$ (1). Chamber was connected to the UNIDOSE digital electrometer (PTW) and the operating voltage was set to 300 volts. All of the measurements were performed inside an automatic MP3 water phantom (MP3-XS, PTW). Chamber positions inside the phantom were automatically controlled using a TBA control unit (PTW).

\subsubsection{Irradiation Setup}

Irradiation in reference condition was considered for output factor measurement and Ksat determination. Dosimetry protocol was based on IAEA TRS-398 and the Italian guidelines for quality assurance in $\operatorname{IORT}(9,12)$. The reference condition during the irradiation of ECB and Advanced Markus dosimeters is reported in Table 1.

Table 1. Irradiation Setup in Reference Condition for Output Factor Measurement by ECB and Advanced Markus Dosimeter

\begin{tabular}{ll}
\hline Parameter & Reference Condition \\
\hline Source to surface distance (SSD) & $71.3 \mathrm{~cm}$ \\
\hline Phantom & Water phantom \\
\hline Depth of placement & Depth of maximum dose (Rmax) \\
\hline Number of reading or irradiations & 3 times repetitions \\
\hline
\end{tabular}

Abbreviation: ECB, ethanol chlorobenzene.

To measure the output factor at various energies, both ECB and Advanced Markus dosimeters were placed inside the water phantom and irradiated at the depth of maximum dose for each energy level (Rmax), along the clinical axis of corresponding flat based applicator. Output factor is generally defined as the ratio of dose at Rmax for a given applicator to that of the reference applicator at the same energy (Equation 1) (2, 9, 12).

Output $\quad$ Factor $(E, r)=\frac{D_{w}(E, r, \Phi)}{D_{w}\left(E, r_{r e f}, \Phi_{0^{0}}\right)}$
Where $D_{w}$ is the dose to water, $E$ is the electron energy, $r$ is the diameter of employed applicator, $\mathrm{r}_{\mathrm{ref}}$ is the diameter of reference applicator $(10 \mathrm{~cm})$, and $\Phi$ is the base angle of the employed applicator. Only flat-based applicators with a diameter of 3 to $10 \mathrm{~cm}$ were considered in this study. For each energy level, irradiations were repeated three times. Because of small irregularities in the home-made vials containing the ECB solution, this dosimeter was irradiated in such a way that the electron beam was perpendicular to the vial axis.

The $\mathrm{K}_{\text {sat }}$ of employed ion chamber (Advanced Markus) at different electron energies was practically determined through comparing the response of ion chamber (uncorrected for ion recombination losses) relative to the ECB dosimeter response. The ratio of ECB response to ion chamber response was considered as the $\mathrm{K}_{\text {sat }}$ of employed ion chamber. It should be mentioned that the $\mathrm{K}_{\text {sat }}$ was determined through employing the flat based applicator with $10 \mathrm{~cm}$ diameter.

\subsubsection{Monte Carlo Simulation}

The head of LIAC IORT machine was simulated using BEAMnrc Monte Carlo code and absorbed dose in water phantom was calculated using DOSXYZnrc code. For each simulation, the number of history was selected as $3 \times 10^{8}$. The electron cut-off energy (ECUT) and photon cut-off energy (PCUT) were considered as 0.521 and $0.01 \mathrm{MeV}$, respectively.

The spatial distribution of electron beam on exit window was considered as a Gaussian intensity profile with full width at half maximum (FWHM) of $1 \mathrm{~mm}$. The mean angular spread of electron beam on exit window was selected as $2^{\circ}$. Energy spectrum of hitting electrons on exit window at different energies was supplied by the manufacturer (Sordina, SpA, Italy). To calculate the output factor, a cubic scoring cell with the dimension of $2 \times 2 \times 2 \mathrm{~mm}^{3}$ was placed at the depth of maximum dose inside a $25 \times 25 \times 25$ $\mathrm{cm}^{3}$ water phantom.

\subsubsection{Statistical Analysis}

Significance test (Independent samples T test) was employed to statistically analyze the difference between the obtained results. In all tests, the significance level was set to 0.05 (Tests with P value $>0.05$ were scored as not significant).

\section{Results}

\subsection{Determination of Recombination Correction Factor (Ksat)}

The recombination correction factors $\left(\mathrm{K}_{\mathrm{sat}}\right)$ determined by ECB, Laitano formalism and standard TVA method (TRS-398) are presented in Table 2. 
Table 2. Recombination Correction Factors Determined by ECB, Laitano formalism and TRS-398 Protocol

\begin{tabular}{|c|c|c|c|c|c|c|c|}
\hline \multirow[t]{2}{*}{ Energy (MeV) } & \multicolumn{6}{|c|}{ Advanced Markus ion Chamber } & \multirow{2}{*}{$\begin{array}{c}\text { ECB Dosimeter } \\
K_{\text {sat }} \text { ECB } \\
\end{array}$} \\
\hline & $V_{1}(v)$ & $\mathbf{V}_{2}(\mathbf{v})$ & $M_{1}(\mathrm{nC})$ & $\mathbf{M}_{2}(\mathrm{nC})$ & $\mathbf{K}_{\text {sat }}$ Laitano & $K_{\text {sat }}$ TRS-398 & \\
\hline 6 & 100 & 300 & 1.910 & 1.896 & 1.001 & 1.004 & 1.002 \\
\hline 8 & 100 & 300 & 1.862 & 1.828 & 1.002 & 1.009 & 1.003 \\
\hline 10 & 100 & 300 & 2.150 & 2.071 & 1.004 & 1.019 & 1.004 \\
\hline 12 & 100 & 300 & 3.651 & 3.495 & 1.005 & 1.026 & 1.006 \\
\hline
\end{tabular}

Abbreviation: ECB, ethanol chlorobenzene.

As reported in Table 2, the $\mathrm{K}_{\text {sat }}$ obtained from Laitano formalism are in very good accordance with those practically derived from ECB dosimetry (lower than $1 \%$ ). No meaningful difference was observed between the results of Laitano formalism and ECB based dosimetry (P value $=0.66$ ). On the other hand, the recombination correction factors calculated by TVA method are considerably higher than those of Laitano formalism or ECB dosimetry (the difference between the results was significant, $P$ value $=0.01$ ) Therefore, employing the standard TVA method during absolute dosimetry of intraoperative electron beam can overestimate the absorbed dose.

\subsection{Determination of Output Factor}

The output factor values for different combinations of electron energy/applicator size determined by ECB, Advanced Markus (according to the Laitano formalism) and Monte Carlo simulation, are shown in Figures 1 - 3, respectively.

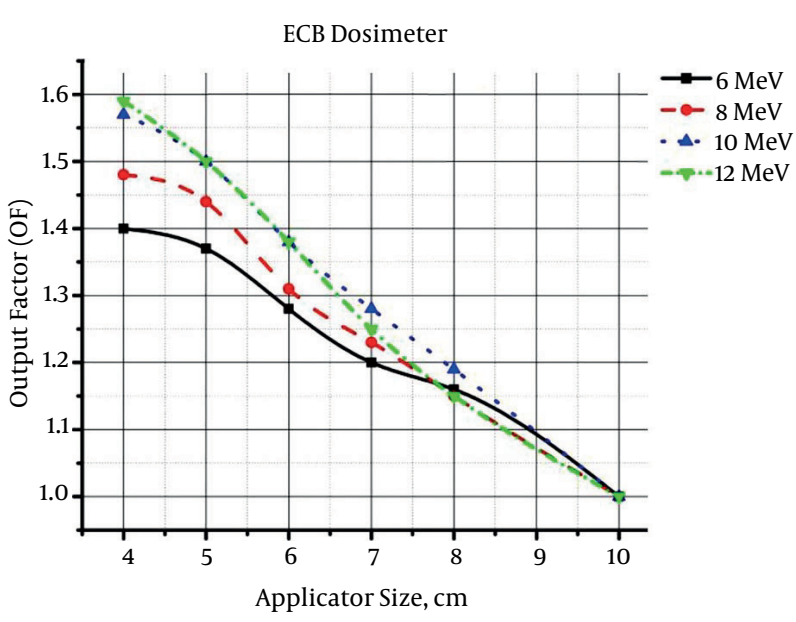

Figure 1. Output factor values at different energy and applicator sizes measured by ethanol chlorobenzene (ECB) dosimeter

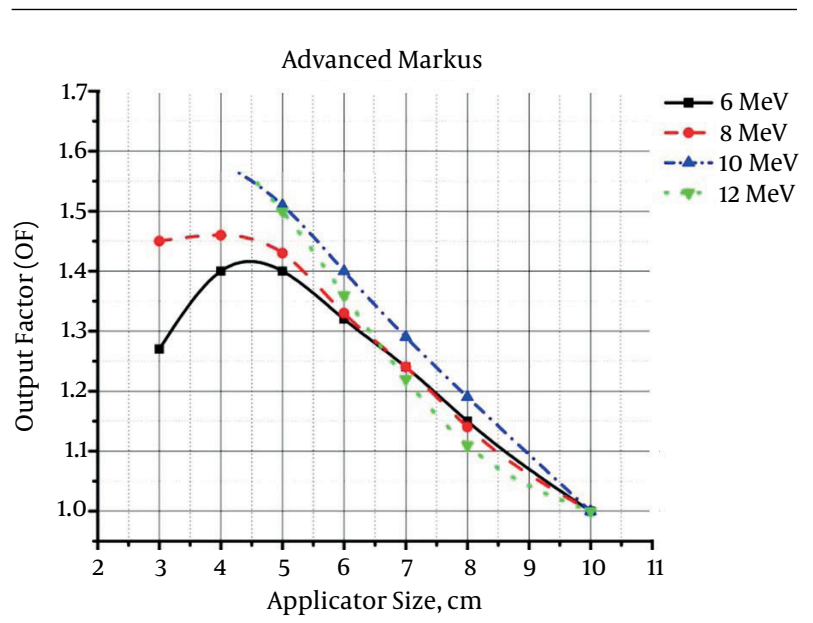

Figure 2. Output factor values at different energy and applicator sizes measured by Advanced Markus ion chamber

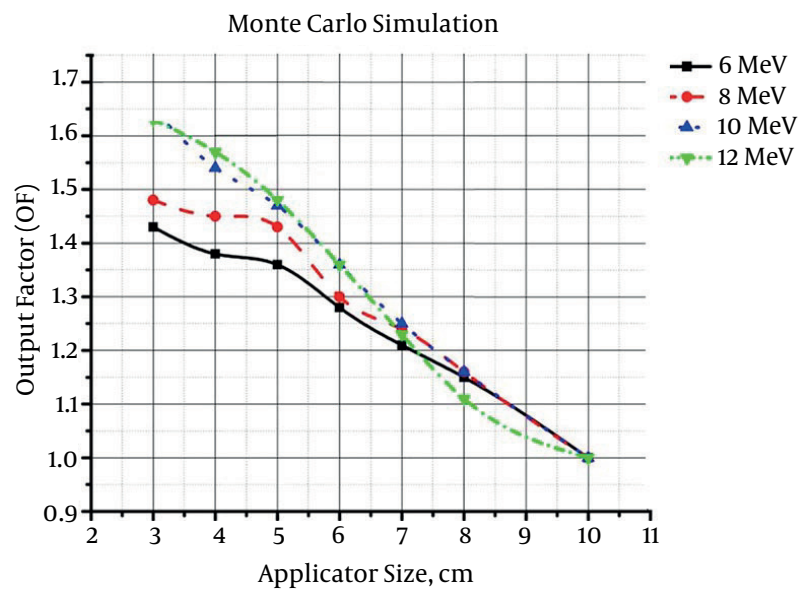

Figure 3. Output factor values at different energy and applicator sizes calculated by Monte Carlo simulation

Due to the large size of employed ECB vials, there were limitations in the output measurement of $3 \mathrm{~cm}$ diameter 
applicator. Therefore, the results of ECB based output factor measurement were reported for $4 \mathrm{~cm}$ diameter applicator and greater ones.

From the presented figures, it could be concluded that by increasing the field size at the same energy, output factor would decrease. On the other hand, by increasing the electron energy at the same field size, output factor increases. By increasing the field size, the energy fluence within the radiation field decreases. As a consequence, the absorbed dose and output factor decreases. Increment of beam energy can increase the energy fluence. This increase in energy fluence would increment the output factor.

The mean relative difference between the output factors measured by ECB dosimeter, Advanced Markus chamber, and Monte Carlo (MC) simulation at different energies are listed in Table 3.

According to Table 3, there is a good accordance between the results of output factor measurement by three different methods. The maximum difference between the results of ECB dosimetry and ionometric dosimetry (Advanced Markus) was equal to $1.5 \%$. On the other hand, the maximum difference between the results of ECB dosimetry and Monte Carlo simulation was $1.7 \%$. As reported by Table 3 , there is no significant difference between the results obtained by three different dosimetry methods (in all cases, $\mathrm{P}$ value is greater than 0.8 ).

\section{Discussion}

In a study performed by De Angelis et al. (12), Fricke and alanine dosimeters were employed for measuring the output factor of a dedicated IORT accelerator. The results of mentioned study showed that the output factors measured by the two dosimetry systems are in a good agreement (the difference between the results was within $2 \%$ ). This difference is comparable with those reported in our study (Table 3 ).

In another study, the output factor of intraoperative electron beam was measured by Gafchromic external beam therapy (EBT) film (23). Due to the high spatial resolution, weak energy dependency, and near tissue equivalence composition have obtained a great importance in the field of radiation dosimetry (23). The results of this study showed that the associated uncertainties with the developed film dosimetry system is equalb to $2 \%$, which was in accordance with the uncertainty reported for ECB dosimeter in the present study (1.5\%).

In a study reported by Iaccarino et al. (24), the output factor for different combination of intraoperative electron energy/applicator size was determined through ionometric dosimetry and Monte Carlo simulation. The results of the mentioned study showed that the mean difference between the two dosimetry approaches is lower than $2 \%$, which is in a good accordance with those reported in our study.

In summary, a novel dosimetry system based on ECB chemical dosimeter was developed in this study to measure the output factor of high dose per pulse intraoperative electron beam. It was the first time that employing such chemical dosimetry system was introduced for output factor measurement of dedicated IORT accelerators and intraoperative electron beam calibration. The validity of ECB based dosimetry results was confirmed through comparing with the results of ionometric dosimetry (Advanced Markus ion chamber) and Monte Carlo simulation. This desirable agreement between the results shows that the ECB dosimeter could be considered as a useful and reliable tool for calibration and output factor measurement of intraoperative electron beam. The main advantage of ECB dosimeter relative to the ion chamber dosimeter is dose per pulse independent response. This feature can significantly simplify the dosimetry process through eliminating the further consideration which should be taken into account for correction of the dosimeter response (e.g. $\mathrm{K}_{\mathrm{sat}}$ which is considered for correction of the ion chamber response). Although studies on usability of ion chambers for calibrating the high dose per pulse intraoperative electron beam still continue, the findings of this study showed that the ECB dosimeter could be considered as a substitution for intraoperative electron beam calibration. Furthermore, the results of this study demonstrated that the chemical ECB dosimeter could be employed as a reference dosimeter for cross calibrating the response of ionization chamber and determination of its recombination correction factor $\left(\mathrm{K}_{\mathrm{sat}}\right)$.

The greatest limitation of ECB dosimeter was its relatively large volume. Due to the presence of high dose gradient regions in small radiation fields, the large size of this dosimeter can affect its usability in such small fields.

In addition, comparing the results of chemical, ionometric and Monte Carlo based dosimetry also showed that the Monte Carlo simulation is a fast and reliable tool for theoretical calibration and output measurement of dedicated intraoperative radiotherapy accelerators. 
Table 3. Mean Relative Difference (\%) Between the Output Factors Measured by ECB Dosimeter, Advanced Markus Chamber and Monte Carlo Simulation

\begin{tabular}{lcccc}
\hline Energy, MeV & Mean Relative Difference (ECB, MC) & PValue & Mean Relative Difference (ECB, Adv. Markus) & P Value \\
\hline $\mathbf{6}$ & 0.6 & 0.962 & 0.853 \\
$\mathbf{8}$ & 0.9 & 0.954 & 0.9 & 0.947 \\
$\mathbf{1 0}$ & 1.7 & 0.848 & 0.6 & 0.975 \\
$\mathbf{1 2}$ & 1.6 & 0.878 & 0.891 \\
\hline
\end{tabular}

Abbreviations: ECB, ethanol chlorobenzene; MC, Monte Carlo.

\section{Acknowledgments}

The authors wish to thank Khatam hospital for providing technical support, Tehran University of Medical Science for financial and material supports and also Atomic energy agency of Iran (gamma irradiation center) for their kind cooperation.

\section{Footnotes}

Authors' Contributions: Seyed Rabi Mahdavi, and Hamid Reza Baghani were responsible for study concept and design. Mojtaba Barzegar, Hamid Reza Baghani, and Seyed Rabi Mahdavi did the data acquisition. Mojtaba Barzegar, Ali Reza Shirazi, and Hamid Reza Baghani performed data analysis and interpretation. Hamid Reza Baghani, and Mojtaba Barzegar carried out manuscript drafting. Mojtaba Barzegar was responsible for statistical analysis. Seyed Rabi Mahdavi, and Ali Reza Shirazi performed administrative technical and material support.

Financial Disclosure: None declared.

Funding/Support: None declared.

\section{References}

1. Baghani HR, Aghamiri SM, Mahdavi SR, Akbari ME, Mirzaei HR. Comparing the dosimetric characteristics of the electron beam from dedicated intraoperative and conventional radiotherapy accelerators. J Appl Clin Med Phys. 2015;16(2):5017. doi: 10.1120/jacmp.v16i2.5017. [PubMed: 26103175].

2. Cella L, Liuzzi R, Salvatore M. The Italian affair: the employment of parallel-plate ionization chambers for dose measurements in high dose-per-pulse IORT electron beams. Med Phys. 2010;37(6):2918-24. doi: 10.1118/1.3432601. [PubMed: 20632603].

3. Schneider F, Clausen S, Tholking J, Wenz F, Abo-Madyan Y. A novel approach for superficial intraoperative radiotherapy (IORT) using a 50 kV X-ray source: a technical and case report. J Appl Clin Med Phys. 2014;15(1):4502. doi: 10.1120/jacmp.v15i1.4502. [PubMed: 24423847].

4. Nairz O, Deutschmann H, Kopp M, Wurstbauer K, Kametriser G, Fastner $\mathrm{G}$, et al. A dosimetric comparison of IORT techniques in limited-stage breast cancer. Strahlenther Onkol. 2006;182(6):342-8. doi: 10.1007/s00066-006-1580-2. [PubMed:16703290].

5. Veronesi U, Orecchia R, Maisonneuve P, Viale G, Rotmensz N, Sangalli $\mathrm{C}$, et al. Intraoperative radiotherapy versus external radiotherapy for early breast cancer (ELIOT): a randomised controlled equivalence trial. Lancet Oncol. 2013;14(13):1269-77. doi: 10.1016/S14702045(13)70497-2. [PubMed: 24225155].
6. ICRU . Dosimetry systems for use in radiation processing. ; 2008.

7. Piermattei A, Canne SD, Azario L, Russo A, Fidanzio A, Micelit R, et al. The saturation loss for plane parallel ionization chambers at high dose per pulse values. Phys Med Biol. 2000;45(7):1869-83. [PubMed: 10943925].

8. Rogers DW. A new approach to electron-beam reference dosimetry. Med Phys. 1998;25(3):310-20. doi: 10.1118/1.598211. [PubMed: 9547498].

9. IAEA TRS-398. Absorbed Dose Determination in External Beam Radiotherapy. ; 2000.

10. Gerbi BJ, Antolak JA, Deibel FC, Followill DS, Herman MG, Higgins PD, et al. Recommendations for clinical electron beam dosimetry: supplement to the recommendations of Task Group 25. Med Phys. 2009;36(7):3239-79. doi:10.1118/1.3125820. [PubMed: 19673223].

11. Araki F, Kubo HD. Comparison of high-energy photon and electron dosimetry for various dosimetry protocols. Med Phys. 2002;29(5):85768. doi: 10.1118/1.1470208. [PubMed: 12033582].

12. De Angelis C, Soriani A, Benassi M, Onori S. On measuring the output of an IORT mobile dedicated accelerator. Radiat Prot Dosimetry. 2006;120(1-4):221-5. doi: 10.1093/rpd/nci621. [PubMed: 16644963].

13. Righi S, Karaj E, Felici G, Di Martino F. Dosimetric characteristics of electron beams produced by two mobile accelerators, Novac7 and Liac, for intraoperative radiation therapy through Monte Carlo simulation. J Appl Clin Med Phys. 2013;14(1):3678. doi: 10.1120/jacmp.v14i1.3678. [PubMed: 23318376].

14. Di Martino F, Giannelli M, Traino AC, Lazzeri M. Ion recombination correction for very high dose-per-pulse high-energy electron beams. Med Phys. 2005;32(7):2204-10. doi: 10.1118/1.1940167. [PubMed: 16121574].

15. Laitano RF, Guerra AS, Pimpinella M, Caporali C, Petrucci A. Charge collection efficiency in ionization chambers exposed to electron beams with high dose per pulse. Phys Med Biol. 2006;51(24):6419-36. doi: 10.1088/0031-9155/51/24/009. [PubMed: 17148826].

16. Johansson B, Wickman G, Bahar-Gogani J. General collection efficiency for liquid isooctane and tetramethylsilane in pulsed radiation. Phys Med Biol. 1997;42(10):1929-38. [PubMed: 9364588].

17. Moretti E, Malisan MR, Frisano K, Padovani R. Charge Recombination Correction in the Dosimetry by Means Ionization Chambers of a High Dose-per-Pulse Electron Accelerator for Intraoperative Radiation Therapy (IORT). IFMBE Proc. 2009;25(3):205-8. doi: 10.1007/978-3642-03902-7_58.

18. Das IJ, Ding GX, Ahnesjo A. Small fields: nonequilibrium radiation dosimetry. Med Phys. 2008;35(1):206-15. doi: 10.1118/1.2815356. [PubMed: 18293576].

19. Pimpinella M, Guerra AS, La Civita S, Laitano RF. Procedure for absorbed dose to water determination in high energy photon and electron beams by ferrous sulphate dosimeter at INMRI-ENEA. Absorbed Dose and Air Kerma Primary Standards. Paris. .

20. IAEA . Standard practice for use of the Ethanol-Chlorobenzene dosimetry system1. ; 2012. 
21. Muir B, Rogers D, McEwen M. Sci-Thur PM: YIS - 07: Monte Carlo simulations to obtain several parameters required for electron beam dosimetry. Med Phys. 2012;39(7):4623. doi:10.1118/1.4740104.

22. Lamanna E, Gallo A, Russo F, Brancaccio R, Soriani A, Strigari L. In: Modern practices in radiation therapy. Natanasabapathi G, editor. ; 2012. Intra-Operative radiotherapy with electron beam.

23. Sorriaux J, Kacperek A, Rossomme S, Lee JA, Bertrand D, Vynckier S, et al. Evaluation of Gafchromic(R) EBT3 films characteristics in therapy photon, electron and proton beams. Phys Med. 2013;29(6):599-606. doi: 10.1016/j.ejmp.2012.10.001. [PubMed: 23107430].

24. Iaccarino G, Strigari L, D’Andrea M, Bellesi L, Felici G, Ciccotelli A, et al. Monte Carlo simulation of electron beams generated by a $12 \mathrm{MeV}$ dedicated mobile IORT accelerator. Phys Med Biol. 2011;56(14):4579-96. doi: 10.1088/0031-9155/56/14/022. [PubMed: 21725139]. 